\title{
Pioglitazone increases risk of bladder cancer
}

A retrospective study of patient records from 600 GP practices in the UK has established a link between use of pioglitazone for type 2 diabetes and bladder cancer risk. All incident cases of bladder cancer occurring after the first year of follow-up $(n=376)$ were matched to multiple controls $(n=6,699)$, demonstrating a mean 1.83 -fold increased rate of bladder cancer in pioglitazone users. This rate approached twofold in patients who took the drug for more than 2 years or received a high cumulative dose. For some clinicians, this finding will come as no surprise. An association between pioglitazone and bladder cancer has been investigated previously-albeit with conflicting findings. In 2011, the FDA and Health Canada added warnings to the drug monograph, but the European Medicines Agency (EMA) decided to maintain marketing authorization.

"Although bladder cancer is a rare condition, we think that physicians and patients need to be aware of the potential risk," recommends Laurent Azoulay, who led the study. "The EMA should join the FDA and Health Canada in adding warnings to the product monograph."

In light of this new evidence, the study authors recommend against prescribing pioglitazone to individuals with a family history of bladder cancer, a history of bladder conditions such as cystitis, or evidence of haematuria. "There are other therapeutic options that can be used in these individuals," says Azoulay.

\section{1 ...increased risk observed with pioglitazone could result from chronic bladder irritation due to crystal formation... 77}

However, despite this latest study, the mechanism behind the link between pioglitazone use and bladder cancer is still unknown. "It is possible that the increased risk observed with pioglitazone could result from chronic bladder irritation due to crystal formation, rather than PPAR-related signalling," hypothesizes Azoulay.

This theory is supported by the lack of a reported association between rosiglitazone (another antidiabetic thiazolidinedione) and bladder cancer. "Although the chemical structures of rosiglitazone and pioglitazone are similar, the doses required to achieve a similar reduction in blood glucose levels are much higher for pioglitazone than for rosiglitazone," Azoulay explains. Further studies-for example, comparing bladder cancer risk with urinary crystal formation-could help to elucidate the mechanism by which pioglitazone exerts its carcinogenic effect.

\section{Melanie Clyne}

Original article Azoulay, L. et al. The use of pioglitazone and the risk of bladder cancer in people with type 2 diabetes: nested case-control study. BMJ doi:10.1136/bmj.e3645 